\title{
ORIENTATION DISTRIBUTIONS OF LOW SYMMETRY POLYPHASE MATERIALS USING NEUTRON DIFFRACTION DATA: APPLICATION TO A ROCK COMPOSED OF QUARTZ, BIOTITE AND FELDSPAR
}

\author{
D. CHATEIGNER ${ }^{\mathrm{a}, \mathrm{b}, *}$, H.R. WENK ${ }^{\mathrm{b}, \mathrm{c}}$ and M. PERNET ${ }^{\mathrm{c}}$ \\ ${ }^{a}$ Lab. Physique de l'Etat Condensé, Univ. du Maine-Le Mans, BP535 \\ F-72085 Le Mans, France; ${ }^{b}$ Department of Geology and Geophysics, \\ University of California, Berkeley, CA 94720, USA; ${ }^{\mathrm{c}}$ Lab. de \\ Cristallographie-CNRS/UJF, BP166 38042 Grenoble Cedex 9, France
}

The crystallographic texture of the three main constituting phases of a granodioritic rock have been determined quantitatively, using neutron diffraction with a position sensitive detector. Intensities deconvoluted from the full profiles were used in the orientation distribution refinements using the WIMV algorithm. Satisfactory results were obtained for the three phases.

Keywords: Neutron diffraction; PSD; Polyphase; Quartz; Biotite; Feldspar

\section{INTRODUCTION}

Rocks which are composed of more than one phase are the most common types in the Earth's crust and in the mantle. Most investigations of the mechanical behavior of rocks have treated them as homogeneous, not considering that the various phases have different grain shapes and a different orientation distribution. Nevertheless, crystallite orientations have a strong influence on deformations which occur during geological processes (Leiss, 1996). Also the interaction of

\footnotetext{
* Corresponding author.
} 
different phases may be important. Unfortunately, the texture of polyphase materials is inherently difficult to determine, particularly for geological samples inside with low crystal symmetries. Using punctual detectors it is difficult to resolve overlapping peaks, with intensity ratios which can strongly deviate from that of a random powder, since all component peaks are not maximum for the same detector position. Using X-rays causes further statistical disadvantages because of the too small probed volume relative to the usual analyzed phases in that kind of samples.

The combination of neutron diffraction and a Position Sensitive Detector (PSD) was indicated several years ago to overcome these problems (Bunge et al., 1982). However no quantitative OD analysis was mentioned in this work. We recently applied the same experimental technique on superconducting materials (Chateigner et al., 1997), and showed it could be used efficiently to resolve multiple Orientation Distributions (OD). It was demonstrated that the determination of two ODs for two orthorhombic phases with significantly different lattice parameters and texture strengths, was possible with a good reliability. The aim of this work is to demonstrate that this method can be applied to even more complex samples. As an illustration, we chosed a granodioritic sample, containing three main phases with trigonal, monoclinic and triclinic crystal symmetries.

\section{EXPERIMENTAL}

\section{Sample Description}

The sample chosen for this work is a mylonitic rock, granodioritic in composition, from the upper part of Palm Canyon south of Palm Spring, California. Results from previous work show that this rock has been deformed in the Santa Rosa mylonite zone during the late Cretaceous (Erskine and Wenk, 1985), in a predominantly ductile mode, with progressive grain size reduction. The aim of this paper being devoted only to the texture methodology, more geological interpretations could be found elsewhere (Wenk and Pannetier, 1990; Wenk, 1998). Our mylonite sample (PC82) was cut in a rounded $1 \mathrm{~cm}$ edged cube for diffraction experiments. Table I shows the constitutive phases 
TABLE I Normative composition of the studied mylonite (PC82, geographic location: $116^{\circ} 30^{\prime} 7.5^{\prime \prime} \mathrm{W} ; 33^{\circ} 34^{\prime} 50^{\prime \prime} \mathrm{N}$. Some traces of epidote and sphene are not taken into account in this table, nor in the calculations

\begin{tabular}{lccccc}
\hline PC 82 mylonite & Biotite & Quartz & Albite & Anorthite & K-spar \\
\hline $\begin{array}{l}\text { Composition } \\
\text { (weight \%) }\end{array}$ & 9.0 & 24.2 & 31.7 & 17.4 & 14.1 \\
\hline
\end{tabular}

and their relative volumes. For this work we decided to consider only the three phases, quartz, biotite and plagioclase, this latter being indexed on the basis of the albite unit-cell. The following crystal systems were assumed: quartz (P32, $a=4.91 \AA, c=5.41 \AA)$; biotite $(\mathrm{C} 2 / \mathrm{m}$, $\left.a=5.3 \AA, b=9.21 \AA, c=10.16 \AA, \beta=99^{\circ}\right)$ and albite $(\mathrm{C} \overline{1}, a=8.14 \AA$, $\left.b=12.79 \AA, c=7.16 \AA, \alpha=94.3^{\circ}, \beta=116.6^{\circ}, \gamma=87.7^{\circ}\right)$.

\section{Experimental Procedure}

We reprocessed experimental data conducted at the D1B instrument of the high flux reactor of the Institut Laue Langevin, Grenoble, equipped with a PSD and a Eulerian cradle as detailed elsewhere (Wenk, 1985). The sample was mounted on the cradle in order to have, after data treatment, the foliation as the projection plane of the pole figures and the lineation parallel to their vertical axis.

The diffracted intensities were recorded in a $2 \theta$ range of $80^{\circ}$, for 500 equally spaced sample orientations. First we summed all the spectra into one, in order to index the complex diffraction patterns for the three main phases. Such a spectrum (Fig. 1) is equivalent to one of a random powder. At this stage we determined the barycenters and half-widths of the individual peaks using a deconvolution procedure (Rodriguez et al., 1992). When the quality of the fit was judged satisfying (correct intensity ratios, coherencies in half-widths, ...) we fixed these parameters. Then, we used them in a cyclic refinement of all 1368 spectra, applying peak deconvolution and integration of intensities, with calculation of the standard deviation on the intensity, based on counting statistics. Special care was taken during this cyclic refinement to exclude negative intensities calculated by the program in some cases. None were observed for the peaks used in the OD calculations, and intensities smaller than two times the standard deviations were rejected. Data were transformed into pole figure coordinates (Bunge et al., 1982) in the Beartex format 


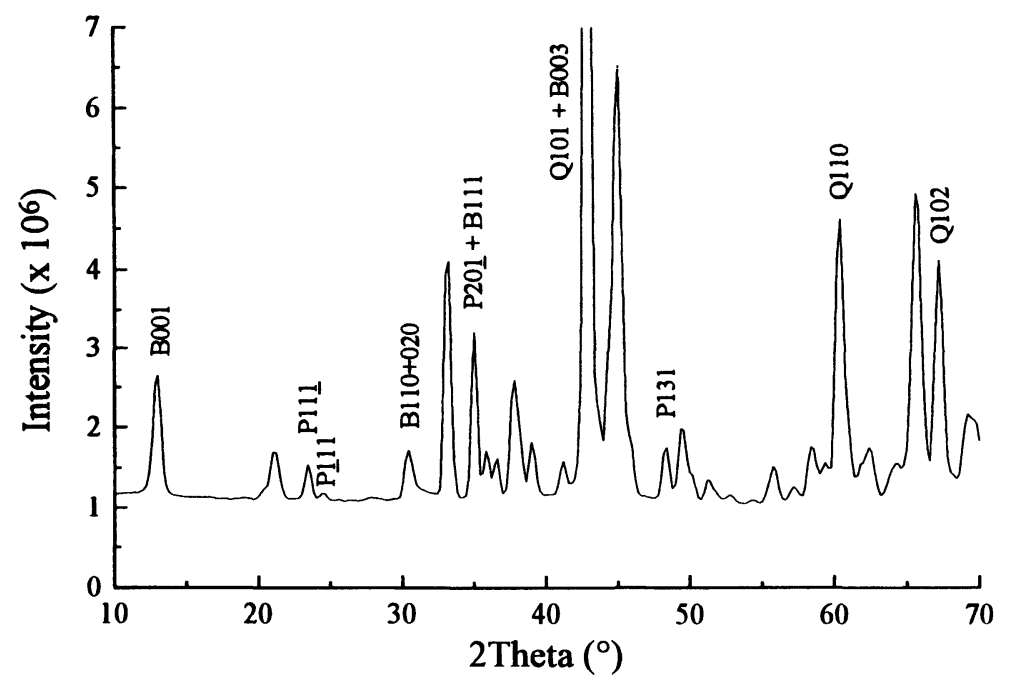

FIGURE 1 Diffraction diagram corresponding to the sum of all measured spectra. $\mathrm{B}$ stands for biotite, $\mathrm{Q}$ for quartz and $\mathrm{P}$ for albite indexing of the plagioclase.

(Wenk et al., 1997) using our local programs. A first normalization of the pole figures was applied at this stage, which was later on refined in the OD calculation step.

Pole figures were constructed for 3 peaks of quartz $(110,102$ and 101), 5 of albite $(\overline{1} 11,1 \overline{1} 1,111,20 \overline{1}$ and 131$)$ and $2(001$ and 020$)$ of biotite. We used the WIMV algorithm (Matthies and Vinel, 1982) implemented in the Beartex package (Wenk et al., 1997) for refining the ODs. We considered several overlaps during this step (see also Table II). For the 102 and 012 reflections of quartz, and the 020 and 110 reflections of biotite. Interestingly, in the case of biotite, the 001 reflection and the $020 / 110$ overlap provide sufficient orientation space coverage as calculated on the MPDS criterion (Helming, 1991). Since we measured complete pole figures, we declared for the refinement the overlap ratio for a random powder.

The estimation of the quality of the OD fit is visually given by the comparison of experimental and recalculated pole figures, and quantitatively by the calculation of the so-called averaged (over the I used pole figures) $\overline{\mathbf{R P}}_{0}, \overline{\mathbf{R P}}_{1}, \overline{\mathrm{Rw}}_{0}$ and $\overline{\mathrm{Rw}}_{1}$ factors (Matthies et al., 1987). The two latter factors are the averaged weighted standard deviations and 
TABLE II Experimental inputs and texture parameters after OD refinement from WIMV

\begin{tabular}{|c|c|c|c|}
\hline & Biotite & Quartz & Albite \\
\hline Used reflections & $\begin{array}{c}010 \\
002+110\end{array}$ & $\begin{array}{c}110 \\
102+012 \\
101+011\end{array}$ & $\begin{array}{c}\overline{1} 11,1 \overline{1} 1, \\
111,20 \overline{1} \\
131\end{array}$ \\
\hline Declared overlaps & $002 / 110$ & $\begin{array}{l}102 / 012 \\
101 / 011\end{array}$ & - \\
\hline OD minima (m.r.d.) & 0 & 0 & 0.1 \\
\hline OD maxima (m.r.d.) & 11.3 & 12.1 & 9.9 \\
\hline $\mathbf{S}$ & -0.81 & -0.58 & -0.15 \\
\hline $\mathrm{F}^{2}$ (m.r.d. $\left.{ }^{2}\right)$ & 3 & 2.8 & 1.3 \\
\hline$\overline{\mathrm{RP}}_{0}(\%)$ & 2 & 9 & 2.3 \\
\hline$\overline{\mathrm{RP}}_{1}(\%)$ & 1.2 & 5.9 & 2 \\
\hline$\overline{\mathrm{Rw}}_{0}(\%)$ & 1.3 & 4.8 & 1.5 \\
\hline$\overline{R w}_{1}(\%)$ & 1.2 & 3.7 & 1.5 \\
\hline
\end{tabular}

were calculated from the individual ones using

$$
\overline{\mathrm{Rw}}_{x}=\frac{1}{\mathrm{I}} \sum_{i=1}^{\mathrm{I}} \mathrm{Rw}_{x}\left(\mathbf{h}_{i}\right)
$$

where $x$ is chosen as 0.05 m.r.d. or 1 m.r.d. respectively, and with

$$
\operatorname{Rw}_{x}\left(\mathbf{h}_{i}\right)=\left(\frac{\sum_{j=1}^{\mathrm{J}}\left[w_{i j}^{\mathrm{o}} I_{\mathbf{h}_{i}}^{\mathrm{o}}\left(\mathbf{y}_{j}\right)-w_{i j}^{\mathrm{c}} I_{\mathbf{h}_{i}}^{\mathrm{c}}\left(\mathbf{y}_{j}\right)\right]^{2}}{\sum_{j=1}^{\mathrm{J}} w_{i j}^{\mathrm{o}} I_{\mathbf{h}_{i}}^{\mathrm{o}^{2}}\left(\mathbf{y}_{j}\right)}\right)^{1 / 2}
$$

where $o$ and $c$ denote the experimental and recalculated diffracted intensities $I_{\mathbf{h}_{i}}\left(\mathbf{y}_{j}\right)=\tilde{\boldsymbol{P}}_{\mathbf{h}_{i}}\left(\mathbf{y}_{j}\right) N_{\mathbf{h}_{i}}$ and associated standard deviations $w_{i j}=$ $1 / \sqrt{I_{\mathbf{h}_{i}}\left(\mathbf{y}_{j}\right)}$. The normalization factors are the values refined in the OD calculation step. The use of the Rws is dictated by the observation of their less texture strength dependency, compared to RPs (Chateigner et al., to be published).

\section{RESULTS}

Figure 2 shows the experimental normalized and recalculated pole figures for biotite (Fig. 2(a)), quartz (Fig. 2(b)) and albite (Fig. 2(c)). All 
(a)
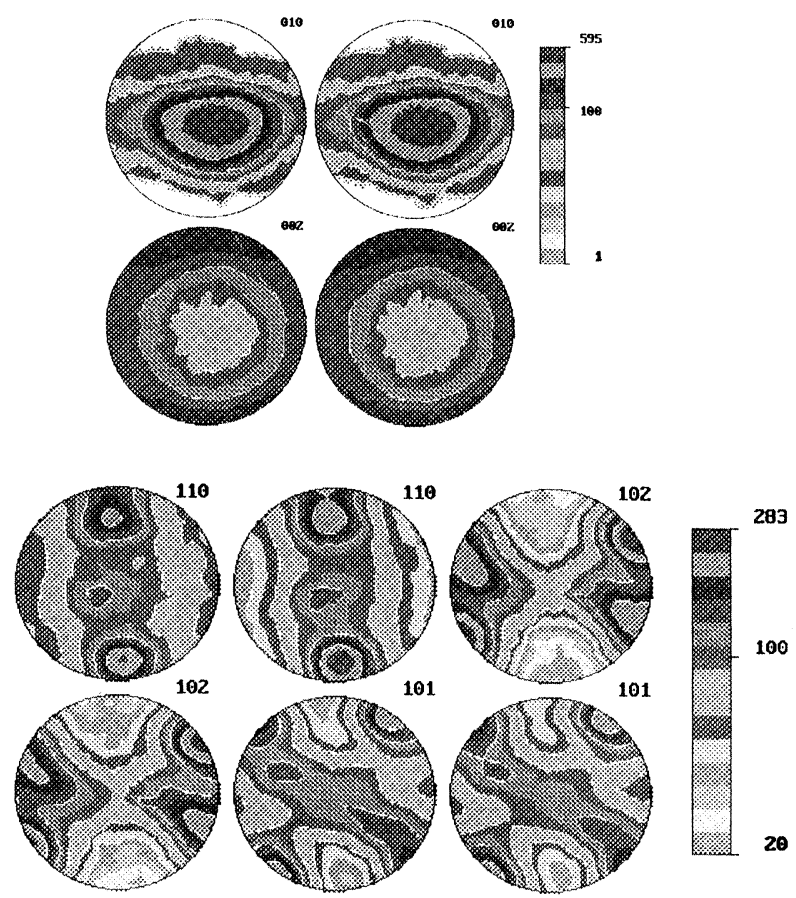

(b)
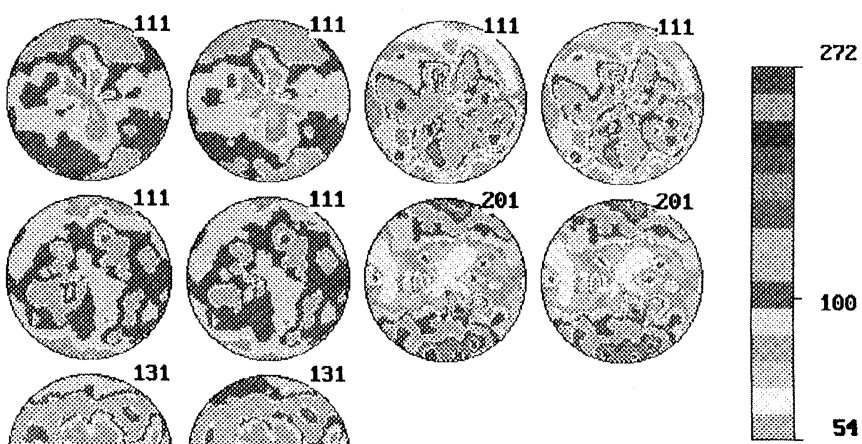

(c)
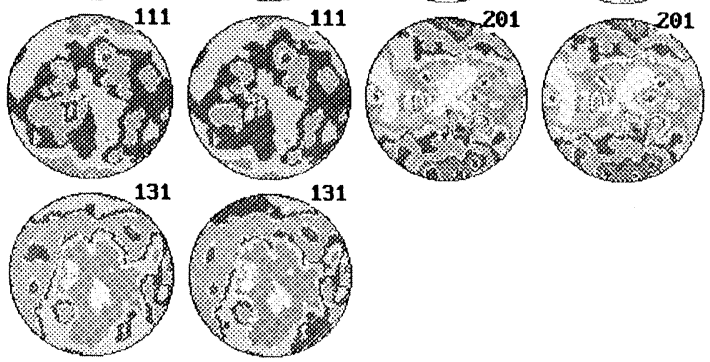

54

FIGURE 2 Experimental-normalized and WIMV-recalculated pole figures in a successive way for biotite (a), quartz (b) and albite (c). Plane of projection is the foliation, lineation is the vertical direction. Equal area projections, logarithmic density scale. The $1 \mathrm{~m} . \mathrm{r} . \mathrm{d}$. level is normalized to 100 . 
figures are found correctly reproduced for the three phases, even the lower level contours. Biotite $\{001\}$ pole figure exhibits a maximum slightly deviated from the foliation normal. It reaches 5.95 m.r.d., indicative of a strong preferred orientation of this phase. This maximum is elongated in the plane perpendicular to the lineation direction. The $020 / 110$ overlap is maximum in the foliation plane with radial constraints. It shows a slight but significant maximum in the lineation direction (more visible on Fig. 3(a), $\{100\}$ pole figure). Due to this

(a)
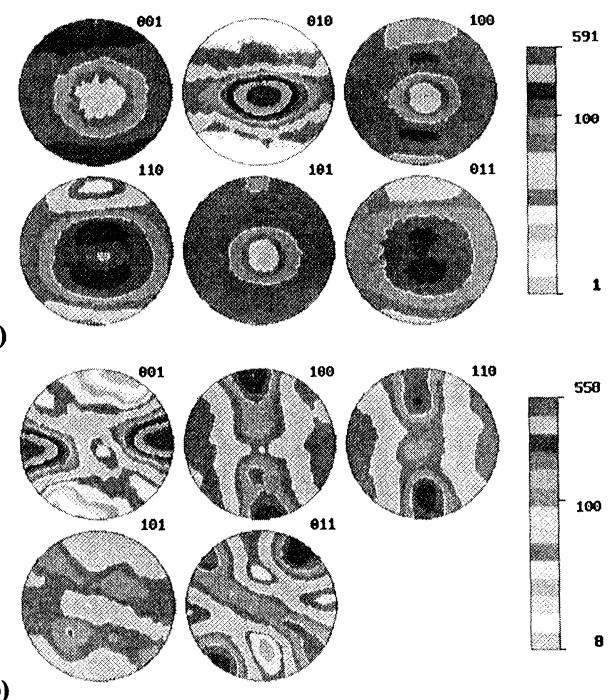

(b)
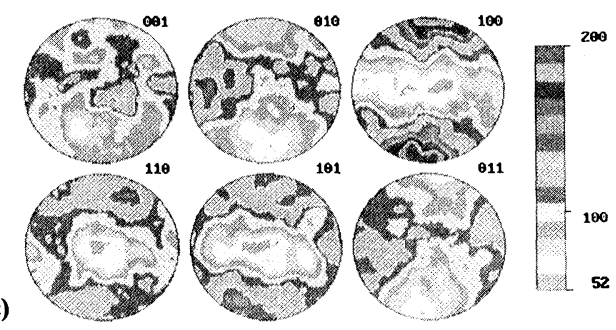

(c)
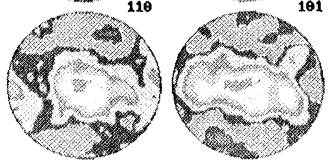

100

.

FIGURE 3 WIMV-recalculated pole figures for low indices reflections, for biotite (a), quartz (b) and albite (c). Plane of projection is the foliation, lineation is the vertical direction. Equal area projections, logarithmic density scale for biotite and quartz, linear for albite. The 1 m.r.d. level is normalized to 100 . 
overlap, we can't directly determine the individual $\{100\}$ and $\{010\}$ pole figures without calculating the OD.

Quartz(Fig. 2(b)) exhibits also a strong three dimensional texture, with a 001 maximum at approximately $90^{\circ}$ from the lineation in the foliation plane, as implied from the $\{110\}$ pole figure. This latter shows the typical 'single crystal' texture (Bunge and Wenk, 1977).

In the case of plagioclase (Fig. 2(c)) the triclinic crystal system makes a direct interpretation of experimental pole figures very difficult, due to their indices. The OD refinement is absolutely necessary if one wants to quantitatively compare samples, but this is delicate when dealing with such a low crystal symmetry.

Table II summarizes the texture informations and calculated parameters after OD refinement for the three phases, maxima in the OD, texture index $F^{2}$, entropy $S$, averaged RP and Rw factors. The reliability factors are satisfactory, as low as $1.2 \%$ for biotite and $1.5 \%$ for plagioclase, as an indication of the good data quality. No large difference is observed between $R_{0}$ and $R_{1}$ factors (RPs or Rws), showing a quite equivalent reliability for all density levels for these two phases. In the case of quartz, $\mathrm{R}$-factors are larger, up to $9 \%$ for $\mathrm{RP}_{0}$. This is attributed to poorer grain statistics.

After refinement of the OD, a strong maximum of the quartz $c$-axes up to $5.6 \mathrm{~m}$.r.d. (Fig. 3(b)) is found at $90^{\circ}$ from the macroscopic lineation, in the foliation plane, with $a$-axes aligned with the lineation. The clear separation of the 101 and 011 poles further evidences the 'single component' texture of this phase.

For biotite (Fig. 3(a)), the slight density deficiency of the $\{100\}$ pole figure along the lineation is an evidence of the alignment of the 010 axes parallel to the lineation direction. The corresponding reinforcement of the $\{010\}$ along this direction is not distinguishable because of the density scale for this representation.

Even if less pronounced, the texture of the plagioclase is documented with a notable $\{100\}$ maximum (at 1.7 m.r.d.) along the lineation, with broad dispersion in the foliation plane. The $\{010\}$ and $\{001\}$ spin more or less freely around $\{100\}$.

The quantitative texture analysis of these three phases allows to characterize their orientational relationships, even independently of the sample position in the geological reference frame, and taking into account their respective dispersions. For the observed maxima of the 
texture components described here, one can deduce these relations:

(100)quartz // (100)albite // (001)biotite $\perp$ lineation

(001)quartz // (100)biotite // foliation

\section{CONCLUSION}

Quantitative texture studies have been largely restricted to single phase materials by conventional techniques. It is now possible to determine textures of polyphase materials using neutron diffraction and peak deconvolution from PSD data. The example presented here shows that texture informations, even contained in complex diffraction spectra of low crystal symmetry polyphase samples, are usable for quantitative analysis.

\section{References}

Bunge, H.J., Wenk, H.-R. and Pannetier, J., 1982. Textures and Microstructures, 5, 153. Bunge, H.J. and Wenk, H.-R., 1977. Tectonophysics, 40, 257-285.

Chateigner, D., Wenk, H.-R. and Pernet, M., 1997. J. Applied Cystallography, 30, 43-48. Chateigner, D., Matthies, S., Lutterotti, L. and Wenk, H.-R. (to be published).

Erskine, B.C. and Wenk, H.-R., 1985. Geology, 13, 274-277.

Helming, K., 1991. Textures and Microstructures, 14-18, 187-192.

Leiss, B., 1996: Geotekt. Forsch., 84: 131 S.

Matthies, S. and Vinel, G.W., 1982. Phys. Stat. Sol. B, 112, K 111.

Matthies, S., Vinel, G.W. and Helming, K., 1987. "Standard Distributions in Texture Analysis", Vol. 1, Akademic Verlag, Berlin.

Rodriguez, J., Anne, M. and Pannetier, J., 1992. STRAP, a System for Time-Resolved Data Analysis (Powder Diffraction Patterns), ILL Grenoble Int. Rept.

Wenk, H.R., Matthies, S., Donovan, J. and Chateigner, D., 1998. J. Appl. Cryst (to appear).

Wenk, H.-R., Heidelbach, F., Chateigner, D. and Zontone, F., 1997. J. Synchr. Radiation, 4, 95-101.

Wenk, H.-R. and Pannetier, J., 1990. J. of Structural Geology, 12(2), 177-184.

Wenk, H.-R., 1985. " Preferred Orientation in Deformed Metals and Rocks: an Introduction to Modern Texture Analysis", H.-R. Wenk (ed.), Academic Press Inc. 